Objectives: To provide an update on opportunistic infections $(\mathrm{OI})$ revised by an independent Safety Adjudication Committee (SAC) (3 paediatric rheumatologists and 2 paediatric infectious disease specialists).

Methods: The participating centres were asked to report all infections encountered by their JIA patients. PRINTO and the medical monitor (MM) classified events based on MedDRA dictionary. Moderate/serious/severe/very severe infections were then revised blindly by the SAC, who was asked to answer 6 questions. The events with consensus of at least $3 / 5$ experts on the first 3 questions ('Is this an infection?', 'Is it common?', 'Is it opportunistic?') were retained for the analysis With referral to the recommendations by Withrop et al. ${ }^{1}$, for the first time a list of Ols in children with JIA on immunosuppressive therapy was elaborated and approved by consensus, through three Delphi steps. Finally, we compared the OI list defined by the SAC to the list of Ol approved by the specialists.

Results: A total of 772 safety events related to 634 patients were submitted to the SAC. $689(89.2 \%)$ events received consensus among the experts on the 3 questions and, of these, $682(99.0 \%)$ were considered as infections, corresponding to 153 different Preferred Terms (PT), according to MedDRA dictionary. Among the 682 infections, $603(88.4 \%)$ were defined by the experts as common and 119 (17.4\%) as opportunistic. For $92(60 \%)$ of the $153 \mathrm{PT}$, the MM and SAC used the same PT, while the remaining $40 \%$ was adjudicated by a third examiner, who analysed again the case reports and assigned the PT which was the most appropriate taking into account the experts' opinion. Among the final number of infections emerged that herpes viral, respiratory and EBV infections were the most frequent (Table). Analysing the infections by PT ( $n=149)$, the experts adjudicated: 22 as OI, 119 as not OI, 8 discordant. Comparing the experts' adjudication with the approved list of OI by PT, there was full agreement for the 22 PT classified as OI, while 19/117 (16.2\%) PT resulted in the list, but were not classified as OI by the experts.

Abstract SAT0386 - Table 1. The most frequent HLT for the 682 infections with agreement of at least $3 / 5$ experts on the fist questions. (N: number of infections)

\begin{tabular}{lcc}
\hline \multicolumn{1}{c}{ HLT } & N & $\%$ \\
Herpes viral infections & 265 & 38.9 \\
Lower respiratory tract and lung infections & 49 & 7.2 \\
Upper respiratory tract inf ections & 44 & 6.5 \\
Epstein-Barr viral infections & 38 & 5.6 \\
Abdominal and gastrointestinal infections & 32 & 4.7 \\
Tuberculous infections & 29 & 4.3 \\
Bacterial infections NEC & 27 & 4 \\
Infections NEC & 19 & 2.8 \\
Ear infections & 18 & 2.6 \\
Candida infections & 17 & 2.5 \\
Influenza viral infections & 14 & 2.1 \\
Streptococcal infections & 14 & 2.1 \\
Salmonella infections & 9 & 1.3 \\
Urinary tract infections & 9 & 1.3 \\
Cytomegaloviral infections & 8 & 1.2 \\
Molluscum contagiosum viral infections & 7 & 1 \\
Papilloma viral infections & 7 & 1 \\
Sepsis, bacteraemia, viraemia and fungaemia & 7 & 1 \\
Campylobacter infections & 5 & 0.7 \\
Staphylococcal infections & 5 & 0.7 \\
Viral infections NEC & 5 & 0.7 \\
Escherichia infections & 4 & 0.6 \\
Pneumocystis infections & 4 & 0.6 \\
Skin structures and soft tissue infections & 4 & 0.6 \\
\hline & & \\
\hline
\end{tabular}

Conclusions: Our analysis showed a significant number of OI in JIA patients on immunosuppressants and provided an approved document stating the most frequent $\mathrm{OI}$ in children with JIA. The most frequent opportunistic pathogens resulted herpes virus, excluding varicella, and mycobacteria, but the list of definite and probable Ols needs to be validated/revised with the analysis of future datasets.

REFERENCE:

[1] Winthrop, et al. Ann Rheum Dis 2015;74:2107-16.

Disclosure of Interest: None declared

DOI: 10.1136/annrheumdis-2018-eular.4484

\section{SAT0387 \\ DESCRIPTIVE ANALYSIS OF LEISHMANIASIS CASES IN THE FUENLABRADA OUTBREAK (SPAIN) ASSOCIATED WITH THE USE OF BILOGICAL THERAPY}

M.P.M. Ahijado Guzmán ${ }^{1}$, R.M. Veiga Cabello ${ }^{2}$, M. Cantalejo Moreira ${ }^{1}$, J. Ruiz Ruiz $^{3}$, A. Zapatero Gaviria ${ }^{3}$, L. Cebrian Méndez ${ }^{4} .{ }^{1}$ Rheumatology Service, Htal Universitario de Fuenlabrada; ${ }^{2}$ Rheumatology Service, Htal Central de la Defensa; ${ }^{3}$ Internal medicine service, Htal Universitario de Fuenlabrada; ${ }^{4}$ Rheumatology Service, Htal Universitario Infanta Leonor, Madrid, Spain

Background: Protozoan parasites of the genus Leishmania can cause opportunistic infections in patients using anti-tumour necrosis factor (anti-TNF) agents, and some authors recommend serological tests for leishmaniasis before using them. An outbreak of cutaneous and visceral leishmaniasis (CL,VL) occurred in the Fuenlabrada area (Spain) in 2010-2012. Some patients were on anti-TNF treatment.

Objectives: We aimed to explore leishmaniasis related to bilogical therapies in the literature, and to obtain data on leishmaniasis cases in patients using antiTNFs referred to our Department of Infectious Diseases

Methods: Literature search of leishmaniasis cases in patients using biological therapies, and to realise descriptive analysis of the clinical features of patients treated with anti-TNFs in our outbreak.

Results: Cases detected in the literature, included our cases, are listed in table 1. In our outbreak of Leishmaniasis, $127 \mathrm{VL}$ and $194 \mathrm{CL}$ cases were detected. Six patients were using anti-TNFs: 2 presented with VL and were using etanercep (33.3\%) and 4 using other anti-TNFs had $\mathrm{CL}(66,6 \%)$. (table 2) These results do not coincide with the total data (table 1): CL 55.2\%, VL 44.8\%, 17 ADA (44,7\%), 16 IFM (42,1\%), 4 ETN (10.5\%), 1 CTZ (2.7\%). There are not more cases described with another biologic therapies at the present time.

Abstract SAT0387 - Table 1

\begin{tabular}{|c|c|c|}
\hline Patholog $j$ & Cutaneous Leizhmaniasis & Viscera leizhmaniasis \\
\hline RhoumatoidArthrits: & $\triangle A \mathrm{ADA}, 2 \mathrm{IFI}, 1 \mathrm{CTZ}$ & $4 A D A, 4 I F M, 2 E T N$ \\
\hline Psoriasic Afthribs & 4ADA1 IFAI & 1ADA 2ETN \\
\hline Ankylosing spondyfitis & 3IFM, 3ADA & 2IFHA \\
\hline Undiferentox:ed Aythribis & & 1IFM \\
\hline Giant cel arterihis & & 1IFM \\
\hline Psotiazis & IADM, ZIFIA & \\
\hline
\end{tabular}

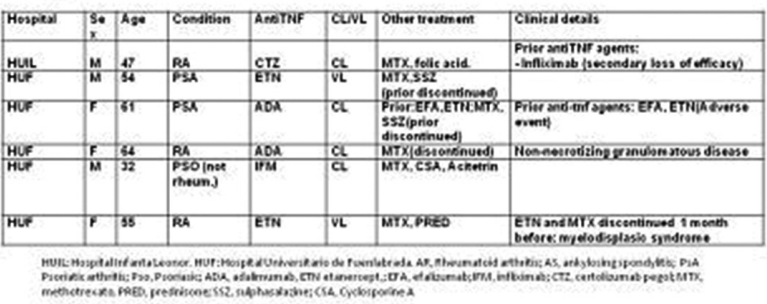

Conclusions: This preliminary detection of leishmaniasis in patients using antiTNFs is aimed at examining the safety of TNF $\alpha$ inhibitors in areas where Leishmania is endemic. This is a small series, but the largest described to date. Our preliminary results suggest that the use of either TNF $\alpha$ receptor or other anti-TNFs has the same probability of triggering the disease but etanercept seems to be associated with fewer, although more severe, cases of VL. Nevertheless, adding all the cases, Table number 1, ADA e IFM are the $86.8 \%$ of the Leishmaniasis, but perhaps will be due to this therapies are combinated and of the most prevalent use. On the other hand, no leishmaniasis cases related to IBD treatment have been described in the literature, suggesting possible involvement of therapies concomitant with the use of anti-TNF with MTX. We detected 3 additional cases of VL and $1 \mathrm{CL}$ using MTX without anti-TNFs. Serological tests for leishmaniasis before using anti-TNFs might not be useful.

\section{REFERENCES:}

[1] Smith VM, Goulden V. Cutaneous leishmaniasis while taking adalimumab for psoriasis. Br J Dermatol [Internet] ((Smith V.M.; Goulden V.) Dermatology Department, Leeds Teaching Hospitals, Leeds, United Kingdom) 2014;171:100.

[2] Guedes-Barbosa LS, Pereira da Costa I, Fernandes V, Henrique da Mota LM, de Menezes I, Aaron Scheinberg M. Leishmaniasis during anti-tumor necrosis factor therapy: report of 4 cases and review of the literature (additional 28 cases). Semin Arthritis Rheum. octubre de 2013;43(2):152-7.

Acknowledgements: To our families

Disclosure of Interest: None declared

DOI: 10.1136/annrheumdis-2018-eular.4165 